\title{
Brain Relevance Feedback for Interactive Image Generation
}

\section{de la Torre Ortiz, Carlos}

ACM

2020-10-20

de la Torre Ortiz , C , Spape , M , Kangassalo , L K \& Ruotsalo , T 2020 , Brain Relevance Feedback for Interactive Image Generation . in UIST '20 : Proceedings of the 33rd Annual ACM Symposium on User Interface Software and Technology . ACM , New York, pp. 1060-1070, Annual ACM Symposium on User Interface Software and Technology , Minneapolis , United States , 20/10/2020 . https://doi.org/10.1145/3379337.3415821

http://hdl.handle.net/10138/328325

https://doi.org/10.1145/3379337.3415821

unspecified

acceptedVersion

Downloaded from Helda, University of Helsinki institutional repository.

This is an electronic reprint of the original article.

This reprint may differ from the original in pagination and typographic detail.

Please cite the original version. 


\section{Brain Relevance Feedback for Interactive Image Generation}

\author{
Carlos de la Torre-Ortiz \\ University of Helsinki \\ Helsinki, Finland \\ carlos.delatorreortiz@helsinki.fi
}

\author{
Michiel M. Spapé \\ University of Helsinki \\ Helsinki, Finland \\ michiel.spape@ @elsinki.fi \\ Tuukka Ruotsalo \\ University of Helsinki \\ Helsinki, Finland \\ tuukka.ruotsalo@helsinki.fi
}

\author{
Lauri Kangassalo \\ University of Helsinki \\ Helsinki, Finland \\ lauri.kangassalo@helsinki.fi
}

\begin{abstract}
Brain-computer interfaces (BCIs) are increasingly used to perform simple operations such as a moving a cursor, but have remained of limited use for more complex tasks. In our new approach to BCI, we use brain relevance feedback to control a generative adversarial network (GAN). We obtained EEG data from 31 participants who viewed face images while concentrating on particular facial features. Following, an EEG relevance classifier was trained and propagated as feedback on the latent image representation provided by the GAN. Estimates for individual vectors matching the relevant criteria were iteratively updated to optimize an image generation process towards mental targets. A double-blind evaluation showed high performance (86.26\% accuracy) against random feedback (18.71\%), and not significantly lower than explicit feedback $(93.30 \%)$. Furthermore, we show the feasibility of the method with simultaneous task targets demonstrating BCI operation beyond individual task constraints. Thus, brain relevance feedback can validly control a generative model, overcoming a critical limitation of current BCI approaches.
\end{abstract}

\section{Author Keywords}

Brain-computer interfaces; Generative models; Image search

\section{CCS Concepts}

-Human-centered computing $\rightarrow$ Human computer interaction (HCI);

\section{INTRODUCTION}

Advances in image generation with neural networks [5, 20, 34] have made it feasible to learn models that are able to generate realistic images that capture semantic structure of the training data. While such models are fascinating in their own right, the

Permission to make digital or hard copies of all or part of this work for personal or classroom use is granted without fee provided that copies are not made or distributed for profit or commercial advantage and that copies bear this notice and the full citation on the first page. Copyrights for components of this work owned by others than the author(s) must be honored. Abstracting with credit is permitted. To copy otherwise, or republish, to post on servers or to redistribute to lists, requires prior specific permission and/or a fee. Request permissions from permissions@ acm.org.

UIST '20, October 20-23, 2020, Virtual Event, USA

(C) 2020 Copyright is held by the owner/author(s). Publication rights licensed to ACM. ACM ISBN 978-1-4503-7514-6/20/10 ...\$15.00.

http://doi.org/10.1145/3379337.3415821

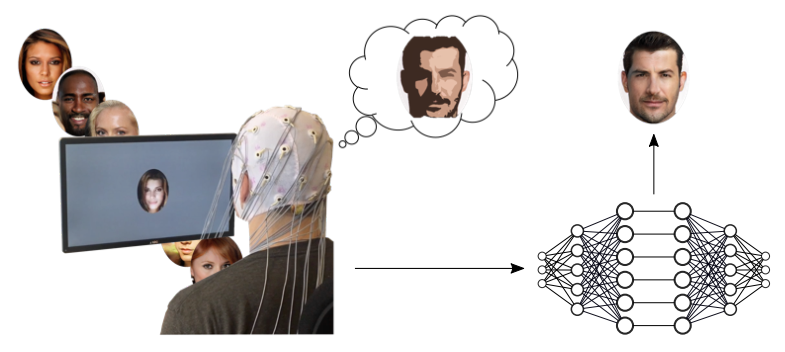

Figure 1. Brain-relevance feedback enables communication between a generative neural network and a human operator via BCI.

practical applications of these models in interactive settings require human interpretation of the learned model. For example, the recent generative adversarial neural network (GAN) models $[8,14]$ are able to generate highly realistic looking images, but controlling the exact features of the generation process remains difficult. Humans, however, are able to describe and detect the features of intended output images quite precisely and often have an "ideal" target image in their mind. Recent advances in brain-computer interfaces have also allowed to infer the human judgements directly from the human cognitive system without requiring any physical activity by the users $[11,12,10]$ and use it as feedback to adapt computation [35, $4,3]$.

Here, we combine a GAN model to rapidly generate highly precise and realistic images, and the human ability to rapidly judge whether the generated images meet the intended goal of the human user, as illustrated in Figure 1. We infer the human judgements toward the images via EEG-based braincomputer interfacing. More precisely, we utilize relevance signal learned from the EEG signal to adjust the output of the GAN model. This allows a neural network to adjust its prediction of the image the user wants to produce by only observing the user's reactions from the user's brain signals. We evaluate the quality of the generated images in response to different types of feedback (brain, random and explicit manual feedback). Furthermore, we measure the amount of feedback required for high-quality image generation by analyzing the 
convergence of the model in response of different amounts of feedback. To this end, we ask the following research questions:

RQ1: Can human brain responses be utilized as feedback to a generative model to produce task relevant images?

RQ2: How fast and well does brain feedback converge compared to explicit and random feedback?

RQ3: How well does brain feedback converge on multiple simultaneous task targets?

In order to answer the research questions, we report two experiments. First, a neurophysiological experiment $(\mathrm{N}=31)$ is conducted in which participants are presented with generated images of human faces that have a distinguishing feature, such as blond hair color, gender, or a smile. Subjects are instructed to focus on a certain feature and to mentally note whenever they see a face displaying the intended feature, while the neurophysiological activity of the subjects is measured via EEG. Then, machine learning models are trained to detect when a subject notices the intended relevant features and the corresponding generated image is used as relevance feedback [32] to adjust a generative model to produce an output image.

Second, a series of simulation experiments is conducted to show the performance of brain-relevance feedback in generating images that match a single task (e.g. generating a smiling face) given to participants in the neurophysiological experiment. To estimate the performance, we analyze the convergence of the models as a function of the amount of feedback. The resulting information can be used to determine the efficacy of this novel type of BCI against random feedback and a high effort scenario in which images are visually classified by human operators.

Third, we test the application of the interface to generate images that fulfill multiple criteria. Even though participants were tasked with a single-criterion mental target (e.g. is the image blond or is the image old), the potential of the interface would be exponentially enhanced if it can generate images beyond the strict constraints of the single type of target.

In summary, our framework is as follows [13]:

1. A generative model produces a realistic image as input for the human operator.

2. The user perceives and reacts naturally to previous input.

3. Relevance is detected from brain responses, updating the estimate position in the GAN latent space.

Note that this is a non-trivial contribution from both a cognitive and a computational point of view: feature conjunction detection is known to qualitatively differ from single feature detection [31] and the high-dimensional GAN space may not efficiently represent combinatorial features.

Thus, by combining brain feedback derived from single-class relevance to generate images fulfilling combinatorial operations (generate a blond, old-looking face, for example), we aim to determine whether such enhanced expressivity is feasible and combinatorial features can be detected.
In summary, our contributions are the following:

1. We present the first of its kind interactive brain-computer interface for image generation combining generative adversarial neural networks and brain feedback.

2. We demonstrate the technique with realistic tasks (such as generate a blond face), and complex combinations of the tasks (such as generate an old female face that is not smiling), and show that the technique is able to generate images matching user intentions.

3. We validate the approach in an off-line study. Our results show performance significantly better than random and approaching manually provided explicit feedback. Our results and generated example images show that our approach yields pragmatic performance suggesting applicability in real-world usage.

\section{BACKGROUND}

Photorealistic synthetic image generation has recently become possible [14]. However, many of the models, while impressive in their generated image quality, remain suboptimal in matching human understanding of the semantic features. That is, the models generate images from random inputs on their latent representations, but the latent representations do not necessarily capture concepts relevant to humans. For example, learning a latent representation of human faces that would learn dimensions directly controlling meaningful features, such as hair color, pose, or age, remains unsolved.

The state of the art GAN architectures, such as StyleGAN architecture [15], tackles this challenge by learning latent features matching "stylistic" features of the images, and thus learn representations that may be closer to the semantic representation that humans might have about the images. However, StyleGAN does still not allow direct control of these features in the generation process and the problem of how to involve human in the parametrization of the latent representation remains uncharted.

Recent research has also aimed to include interactive controls over generative models. For example, a user providing a freely formed sketch of the intended image output for a generative model, which then completes the image by generating it using the shapes and other features present in the sketch [9, 25, 20]. Research has also shown initial approaches for text to photorealistic image synthesis $[36,34]$ in which a user provides a textual description as an input and the system generates an image with the features matching the textual input. While impressive, all previous approaches rely on user control which is implemented for a specific model and approach in advance. That is, the system is trained to detect a certain feature change in the latent space in a supervised manner and then maps the representation space to a custom built user interface control.

An intriguing alternative approach, presented in this article, is an interactive direct interface between the human cognitive system and the generative model. This allows direct readings of the human reactions from the human brain responses and feeds these reactions back to the model to adjust the generation process in the subsequent iterations. 
Human cognition can be characterised as extracting relevant patterns from sensory and stored data for optimising behaviour towards survival. Thus, uncommon, task-related, and informative stimuli are attended to and prioritised in processing over other stimuli, as they are used to adjust our knowledge of the environment [17]. The P300, a positivity in parietal electoencephalographic activity that arises ca. $300 \mathrm{~ms}$ after the onset of relevant stimuli may be a neurophysiological correlate of this process [1] as the $\mathrm{P} 3$ potential is typically evoked in response to improbable, task-relevant, and response-informing events $[22,23,33]$.

As the P300 is one of the most reliable neurophysiological markers of cognitive functioning, it has long been used as an input signal to operate classic brain-computer interfaces (BCIs), such as the BCI-speller [6]. Interfaces like these use a grid of letters in which the different rows and columns are seen as rapidly blinking. The user then concentrates on a letter within a grid, so that during the blinking, the relevant letter is uncommonly (e.g. 1 out 5 rows or columns) present - what is known in the rapid serial visual presentation literature as an "oddball" [2]. This family of BCIs then classify the relevant row and column, typically as evoking the largest P300s, in order to transmit a single command and type the intended letter.

In contrast to the BCI-speller type interfaces that transmit a multi-class (e.g. 1 in 26 different letters) based on a lengthy series of binary (relevant vs. irrelevant) classifications, more recent BCIs aimed to adapt a hypothesised optimal position to feedback signals derived from the ERP. For example, a neuroadaptive interface of this type was able to successfully relate ERPs related to expectation violation to infer intended cursor movements within 2-dimensional space [35]. In the present framework, we similarly infer and generate a type of movement, but instead of employing the manifest, visual space, we estimate the latent direction through the multidimensional feature space, generating images from the generative adversarial network model. That is, we utilize the P300 to mark the presence of relevant features appearing in the generated images. Then, the relevant classifications can be associated with the image features as present in the computer-learned representation of the image space.

Recent brain-computer interfaces have aimed at utilizing generative models similar to our approach $[30,26,16]$. However, their attempts were called into question due to confounds in the experimental design, which explained the effects instead of the computational model [18]. Therefore, adapting generative models using brain feedback has remained an unsolved problem, which we address with a more controlled, randomized experimental design that is derived from the conventional "oddball" methodology, which has a long tradition in EEG research [28].

Unlike previous work, we present a novel technique to support BCI research by developing a brain-based control for generative neural network. In our study, EEG data is obtained from 31 users who viewed ca 2400 GAN-derived images while performing 8 simple feature detection tasks. Using machinelearning, we estimate relevance and demonstrate a generative off-line process able to produce images matching the tasks. Furthermore, we simulated a generation process with multiple criteria based on brain feedback derived from single feature detection tasks. The products of the image generation were then evaluated by external judges who provided objective quality assessment.

\section{METHODS}

Our research relies on the neural signals measured from participants in response to perceiving a generated image. We first describe the neurophysiological experiment used to acquire EEG data from 31 participants in response to eight singlefeature detection tasks. Secondly, we present the model used to generate images and the brain feedback that is used to control it. Then, we show the EEG classification strategy into relevant and irrelevant classes. We follow with a description of the image generation pipeline, presenting the GAN model, feedback updating, and combined task simulation. Finally, we give an account of our strategy used to measure the efficacy of the models for generating relevant content.

\section{Neurophysiological data acquisition}

Volunteers $(\mathrm{N}=31)$ were recruited for the data acquisition part of the study. They were generally undergraduate students from the faculty of Science, 13 were female and 18 male (gender self-reported), and they were $28.23(\mathrm{SD}=7.14$ ) years old on average. The study was approved by the University's Ethical Review Board and conducted in accordance with the Declaration of Helsinki. Accordingly, participants were all fully informed as to the nature and purpose of the study, as well as in regards to their rights, including the right to withdraw at any time without fear of negative consequences. Participants received cinema vouchers in appreciation of their support.

\section{Stimuli and apparatus}

Images were generated using a GAN architecture trained on a of celebrity image dataset [14]. The generative model was sampled via a random process of ca. 10,000 latent vectors from a 512-dimensional multivariate normal distribution, producing pictures of artificially-generated faces. All generated images were manually categorized these into eight study tasks (blond, dark-haired, female, male, old, young, smiling, not smiling), and filtering these to remove visual artifacts. This process resulted in each category having ca. 260 images that were shown to a single participant during the first phase of the experiment. Following, the $1024 \times 1024$ px sized images were standardized by applying a silhouette ellipsoid cut-out of $746 \times 980$, and down-sampling the result by a factor 2 to improve temporal accuracy during data acquisition.

Experiment presentation, hardware synchronization and timing optimization was done using E-Prime 3 [27]. Images were displayed at ca. $60 \mathrm{~cm}$ on a 24 " LCD monitor running a $1920 \times 1080$ resolution at $60 \mathrm{~Hz}$. EEG was obtained using a BrainProducts QuickAmp USB amplifier collecting EEG data at $1000 \mathrm{~Hz}$ with passive $\mathrm{Ag} / \mathrm{AgCl}$ electrodes placed on 32 equidistant scalp locations of the 10/10 system, along with an additional electrode over $\mathrm{AFz}$ acting as ground. 


\section{Procedure}

Participants were instructed to passively observe series of images while keeping a mental selection task in mind. A standard rapid serial visual presentation (RSVP) paradigm with a single-feature recognition task without overt response was used to show randomized sequences of 70 images. These were sampled from the collection of the images expected to have the relevant feature and those expected not to have it (e.g. in the "blond" feature recognition task, images from the blond and dark-haired sets were sampled). Following a reminder of the task (e.g. mentally concentrate on "blond" people), images were shown at a constant pace of $500 \mathrm{~ms}$ per image, followed by a self-terminated break. After 4 RSVP trials (280 images), the relevant category was changed to the next. The order of images was randomized within blocks of 4 trials while the order of relevant categories was counterbalanced between subjects. The data acquisition took around an hour to complete.

\section{EEG pre-processing}

Band-pass EEG data was filtered in the frequency range of $0.2-35.0 \mathrm{~Hz}$ with a Firl filter, removing slow signal fluctuations and high frequency noise. The data were then split into baseline-corrected epochs varying from -200 to $900 \mathrm{~ms}$ relative to stimulus onset. Transient artifacts (e.g. blinks) were removed by heuristic thresholding based on the highest absolute maximum voltage, which resulted in rejecting ca. $11 \%$ of epochs per participant. Finally, the data were decimated with a factor of 4 to decrease classifier training time. The epoched dataset included 3251 epochs per participant on average.

\section{Classification}

During neurophysiology data acquisition, randomly sampled images $(X)$ were presented to the user while their EEG signal $(S)$ was recorded, allowing temporal mapping $X \leftrightarrow S$. Therefore, subsets of $S$ occurred when an image with different features of interest was presented to the user. Differences among these $s_{n}$ allow to train a classifier (target vs. non-target stimulus), providing a classification function $f: S \rightarrow C$, where $C$ is the task-specific classification result for given EEG data.

Consequently, regularized Linear Discriminant Analysis (LDA) classifiers were trained for each of the participants and tasks. Vectorized representations of the ERPs together with class-membership binary labels ("relevant" or "irrelevant") were used to train the classifiers. Every classifier was trained with data gathered during all of the participant's tasks, except the task about which predictions were to be produced and the opposite one. Therefore, a classifier predicting the labels for the "female" task would be trained with data from "blond", "darkhaired", "old", "young", "smile" and "nosmile" tasks. The opposite task was removed from the training set to guarantee there are no brain responses for the same stimuli set in the training and test sets. To minimize the amount of false positives, only predictions with a trust score greater than 0.7 were deemed accurate for the particular class.

After pre-processing data of each participant was stored in a $e \times c \times t$ tensor ( $e$ epochs, $c$ channels, and $t$ sampled time points). Each epoch was split into 7 equidistant time windows $(w)$ on the $50-800 \mathrm{~ms}$ post-stimulus period. The measurements within each time window were averaged and all the available channels and averaged time windows were concatenated to generate spatio-temporal feature vectors. The result is a $n \times$ $(c \cdot w)$ matrix where $c \cdot w=32 \times 7=224$, which was used as an input to train the classifiers.

\section{Image generation}

The neural signals measured from participants in response to perceiving a generated image are classified as relevant or irrelevant. This relevance label is then used as feedback to update an estimate of a target image in the latent space of a GAN model. Here, we describe the model and computations used to generate images based on the classifier output.

\section{Generative model}

The generative model (GAN) provides mapping between the latent space and the stimuli (image) space [8]. GANs are a class of artificial neural networks consisting of a discriminator $D$ and a generator $G$ at training stages. Both are trained simultaneously in a "game" in which $G$ is tasked with producing output indistinguishable from training data. In contrast, $D$ needs to discern whether input images indeed belong to the training dataset or are a $G$ forgery. $G$ and $D$ are therefore engaged in adversarial training until $D$ cannot consistently distinguish training data from $G$ output. At this point, $D$ can be removed and $G$ is considered fully trained. The generative model therefore holds an internal representation (latent) space of training data.

We used a pre-trained GAN to generate face images $[14]^{1}$. This generative model was trained with images of celebrity faces (CelebA-HQ dataset) [14], a higher resolution version of the CelebA dataset [19]. The generative model produces realistic face $1024 \times 1024$ px images from a 512-dimensional latent vector. The GAN thus provides a mapping $G: Z \rightarrow$ $X$, such as $G\left(z_{n}\right)=x_{n}$, where $z_{n} \in Z$ is a point in a latent space $Z$, and $x_{n} \in X$ is digital information perceivable by humans (images of faces). For that, the generator produces images $\left(x_{0}, \ldots, x_{n}\right)$ from vectors $\left(z_{0}, \ldots, z_{n}\right)$ drawn from the latent space.

\section{Relevance feedback and model updating}

The attempt is to find a point $\hat{z}_{n} \in Z$ for which $G\left(\hat{z}_{n}\right)=\hat{x}_{n}$ matches the user's mental target image. We approach this via relevance feedback [37]. The idea is to navigate in the latent space $Z$ of the generator $G$, until they reach the point $\hat{z}$ so that the image $G(\hat{z})$ matches the mental target intent. Positively classified latent vectors $\left(\hat{z}_{n}\right)$ can be used to update the estimate $\hat{z}$, steering the generative model towards task target. That is, we expect that $G\left(\hat{z}_{n}\right)=\hat{x}_{n}$ generation output will be closer to the mental target image than $G\left(\hat{z}_{n-1}\right)$. Further updates of $\hat{z}$ with correct classifications will allow to reach convergence towards user's mental target image.

In detail, the GAN feedback updating strategy is as follows: at every time iteration, a stimulus image is obtained from the generative model from a latent vector. In order to obtain vectors for relevant and irrelevant images, these latent vectors are

\footnotetext{
${ }^{1}$ Source code and pre-trained models: https://github.com/ tkarras/progressive_growing_of_gans
} 
sampled so that those violating the target are overrepresented. This is necessary as the evoked brain potentials for images with relevant features are amplified when presented along with images with irrelevant features.

Now, the latent vector classified as relevant, we can update the estimate with a variant of the well-known Rocchio algorithm [24]. We calculate the average of the vectors of the images classified as relevant and denote the resulting vector by $z_{\text {avg }}$. Then we update the vector $z_{n}$ by letting $z_{n+1}=z_{n}+z_{\text {avg }}$. The location $z_{n}$ should now step-by-step move in $Z$ and eventually approach the point $\hat{z}$ that yields the desired target image $G(\hat{z})$. We chose to use the vectors of the images classified as relevant as positive feedback (moving towards the target) and the vectors of the images classified as irrelevant as negative feedback (moving away from the target).

\section{Combined task simulation}

Generative model outputs were also simulated by combining feedback from several individual tasks. This was done simply by providing relevance feedback in a form of latent vectors $z$ from multiple tasks sequentially. For example, to test a combination "blond" and "male" face features, we first provided latent vectors corresponding to images classified as relevant from a task blond and then from the task male. Combinations containing any opposing individual tasks were excluded (e.g. the combination of "old" and "young") as this would have not led to any meaningful combined task.

\section{Performance evaluation}

We analyzed the performance of each task and participant LDA classifier calculating the Area Under the ROC Curve (ROC). Image generation for each feedback model was evaluated by a single user study. Reliability of this method was further assured determining inter-rated agreement in a smaller study in the same conditions.

\section{Classifier evaluation}

Performance was evaluated via AUC and validated by a permutation test. In the permutation test, permutation-based $p$-values were obtained by contrasting task classifiers AUC scores with those of classifiers trained with randomly permutated class labels [21]. $k=100$ permutations were performed per participant, resulting in a theoretical minimum $p$-value of 0.01 [7].

Classifiers performance was also evaluated as a function of available training data, emulating initial user data calibration.

\section{Image generation evaluation}

The generating pipeline was run for three conditions: brain relevance feedback, explicit feedback, and random feedback. To generate explicit feedback, human-assigned labels were used to determine relevance. In the brain relevance feedback, the classifier output was used. A random process was used to generate images at iteration 29 by random permutation of the relevance labels obtained with brain feedback.

The quality of the generated images was manually assessed by a single user using a randomized evaluation task. Each single image the user was requested to evaluate was randomly drawn from the pool of images generated using explicit feedback, brain-based feedback, or random feedback, with the last functioning as a control condition. To assess the sequential convergence, images were were sampled at iterations $0,9,19$, and 29 after first image presentation in the task. Because there would have been a very large number of unique combinations of two and three tasks, combinations of two and three tasks were randomly sampled so that eight randomly sampled combinations of two and eight randomly sampled combinations of three tasks were used.

To assess the generated images, these were shown along with their associated label, and the user marked the degree of matching on a discrete scale: "no match" (0), "partial match" $(0.5)$, and "total match" (1). As images were shuffled and only the associated category rather than the generative model, the evaluation procedure followed a blind protocol to fairly assess the quality of the models against a random control. The study continued until all generated images were rated. To further evaluate the reliability of the procedure, an additional assessor rated 100 randomly sampled images using the same procedure to determine inter-rater agreement. The Cohen's Kappa of 0.72 shows substantial agreement between raters.

\section{RESULTS}

\section{Neurophysiology}

To verify that relevant features reliably evoked distinguishable patterns in the EEG, we conducted an event-related potential analysis on the average difference between relevant and irrelevant stimuli. As can be seen in Figure 2, a difference between relevant and irrelevant images could be discerned from ca. 250 $\mathrm{ms}$ after inset, growing increasingly large (maximal difference of $2.36 \mu \mathrm{V}$ at $464 \mathrm{~ms}$ ) until well after subsequent stimulus onset (at $500 \mathrm{~ms}$ ). In particular the relevant images evoked an additional, third positive peak over parietal sites at a latency of ca. $380 \mathrm{~ms}$. We furthermore analyzed whether the task affected the pattern and found that latency and amplitude varied, likely in response to task complexity (e.g. the "blond" detection was faster than the "old" detection). However, relevant stimulus categories generally evoked a similar pattern with positivity peaking in the range of 250 and $450 \mathrm{~ms}$. Testing each millisecond of activity within the ERPs evoked by relevant and irrelevant stimuli against one, we found a significant difference starting at ca. $280 \mathrm{~ms}$, and continuing until ca. 700 $\mathrm{ms}$, with a maximal difference observed at $464 \mathrm{~ms}$ over the Pz electrode $(\mathrm{D}=2.36, \mathrm{t}(30)=11.80$, Bonferroni corrected at 1000 comparisons $\mathrm{p}<.00001)$.

In sum, the latency, topography and effects of task-dependence suggest the observed pattern in the event-related potential is consistent with the P3 literature [29, 23].

\section{Classifier performance}

Figure 3 Shows average LDA classifier AUC over all subjects with increasing training data up to an average of 1497 samples. Total samples per participant varies as a result of data pre-processing. Classifier performance increased as function of training data, showing early convergence. Average performance improvements, although smaller, continue up to 


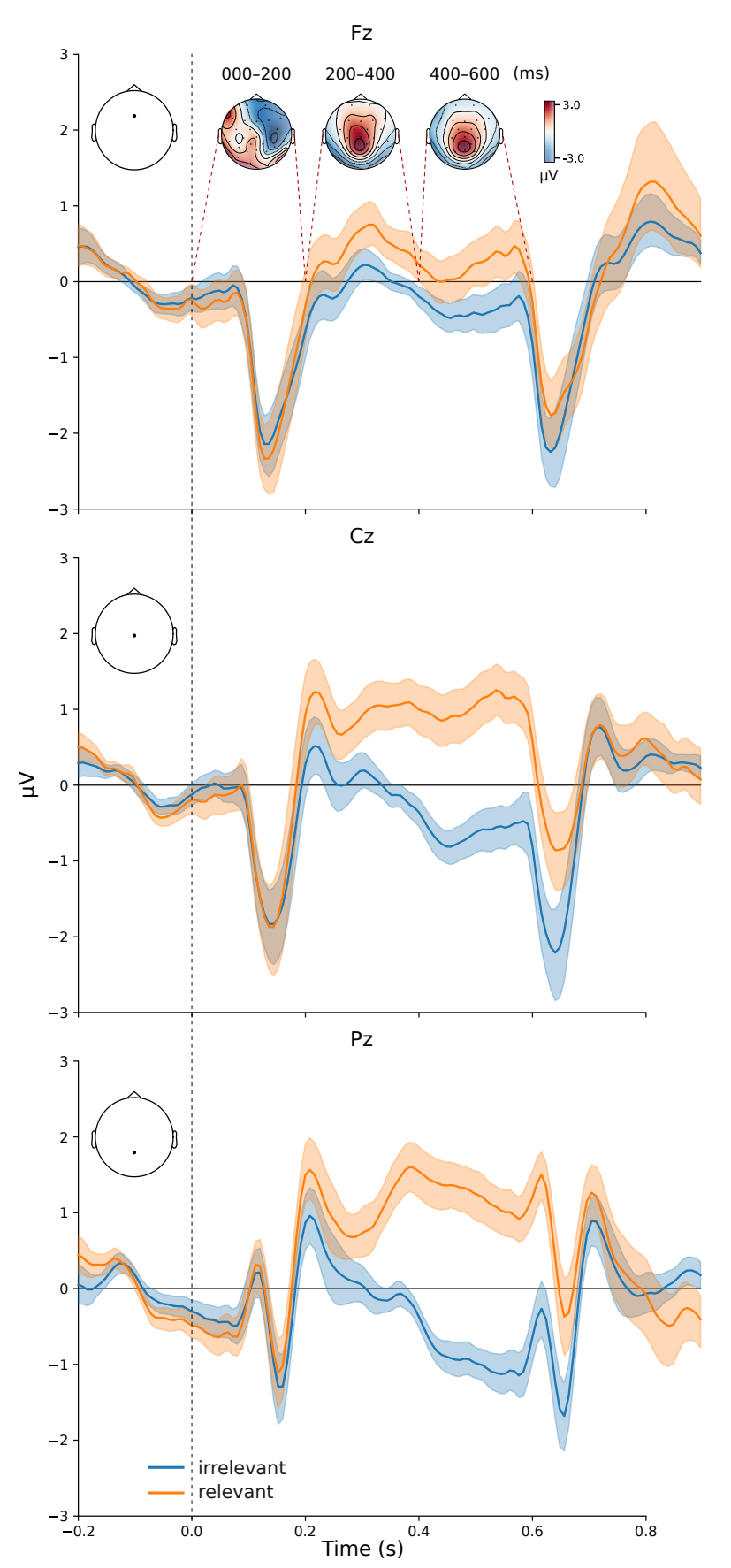

Figure 2. Average ERP plots for all participants (Fz, Cz, and Pz channels) in the -200-800 ms window. Topographic map of the averaged scalp data field values at $0-200,200-400$, and $400-00 \mathrm{~ms}$ post-stimulus windows.

later iterations. Average LDA classifier per participant was analyzed. In all cases, median classifier AUC was above 0.7.

\section{Quality of generated images}

Images were generated at every time iteration from averaged relevant latent vectors up to that iteration. Image pull was sampled down to iterations $0,9,19,29$ to make evaluation possible. Figure 5 shows example generated data for 2 example single tasks, 2 combinations of two tasks, and 2 combinations of three tasks. Images are displayed in iteration sequential order, and for all three feedback models. Each of the 6 task/combinations image blocks with all feedback models represents output for one participant.

Generated images are shown to increasingly match the task as more feedback classified as relevant is available to the generative model (brain and explicit feedback models). For each task experimental block, the opposite task is overrepresented. Therefore, the generative model is more likely to produce opposite task image features at lower iterations and by a random process (random feedback).

\section{Convergence}

Figure 4 shows the generative model performance evolution with the different feedback models: random, brain, and explicit feedback.

With additional relevant feedback provided to the generative model, performance rapidly increases. We can observe near convergence as soon as in ten image presentations (iterations). Convergence is slower as the stimulus gets more complex i.e. more tasks are combined in our experiments. Performance improvements, although smaller, continue up to later iterations.

The quality of image generation was assessed at iterations 0 , 9,19 , and 29 by user rating for the single task and sampled combinations, and all feedback models. At the last iteration we consider full convergence for the single tasks and its combinations.

Table 2 shows all data for tasks performance, significance, and improvement in image quality across feedback models. Applying a one-way ANOVA, image quality score distributions were compared to determine if they were statistically significant. Bonferroni corrected $p$-values and the respective $F$-statistics were obtained. For the single task, image scores for generation using brain feedback were statistically always significant vs.

\begin{tabular}{lrr} 
& \multicolumn{2}{c}{ Performance and Significance } \\
\cline { 2 - 3 } & $A U C$ & $(p) B v s . R$ \\
\hline Single Task & & \\
Female & 0.87 & $2.80 \times 10^{-28}$ \\
Male & 0.82 & $7.14 \times 10^{-23}$ \\
Old & 0.75 & $2.27 \times 10^{-17}$ \\
Young & 0.75 & $3.19 \times 10^{-09}$ \\
Smiles & 0.82 & $7.53 \times 10^{-14}$ \\
Nosmile & 0.78 & $2.61 \times 10^{-11}$ \\
Blond & 0.85 & $2.21 \times 10^{-17}$ \\
Darkhaired & 0.81 & $5.63 \times 10^{-25}$ \\
Combined Tasks & & \\
1 task & 0.81 & $4.02 \times 10^{-10}$ \\
2 tasks & 0.81 & $1.59 \times 10^{-2}$ \\
3 tasks & 0.81 & $1.88 \times 10^{-3}$ \\
\hline AUC per task and
\end{tabular}

Table 1. AUC per task and average of combinations at iteration 29. All results are significant compared to a random classifier (permutation test). 


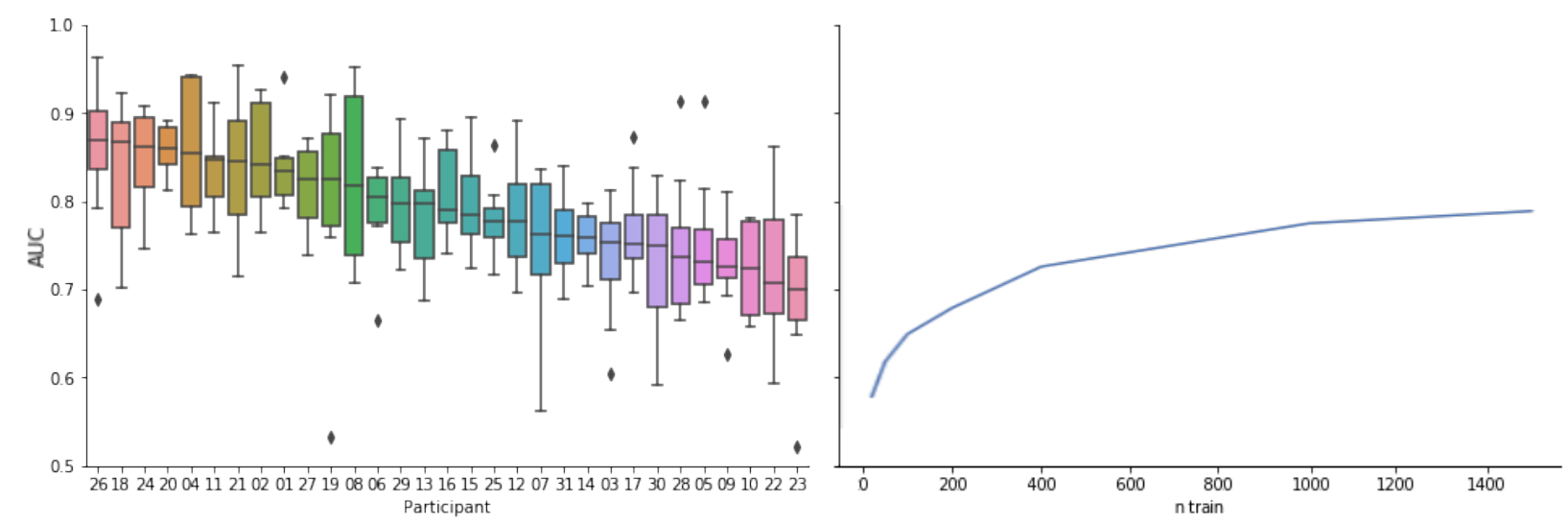

Figure 3. Classifier performance. Left: AUC scores of the classifier for each participant trained with all samples. Right: Averaged and interpolated AUC scores for classifiers trained with $20,50,100,200,400,1000$, and all (1497 on average) samples.

random feedback generation, independently of the specific task. None of the tasks showed statistically significant results when comparing explicit feedback vs. brain feedback.

In the case of the combinations of two tasks, all cases brain vs. random feedback were significant. However, the "male young" combination has a lower $p$-value than the other combinations. This could be explained as the task "young" tends to produce faces of female individuals. In a similar manner, the quality scores difference for the combination "blond male" were statistically significant in the case of brain vs. explicit feedback.

Combinations of three tasks scores are also statistically significant independently of the combination. The effect observed for the "blond male" seems to revert when the "nosmile" task is introduced in the combination, as the task "nosmile" tends to generate more faces of male individuals. The "darkhaired female old" combination has a lower $p$-value than the other combinations. This could be explained as the "female" task produces faces of female individuals, who also tend to be blond and young.

Table 1 summarizes LDA classifier performance (AUC) and generation evaluation score significance. Random and brain feedback models scores are compared showing their $p$-values. In this case, the individual tasks and averaged task combinations are evaluated. For all single tasks and combinations, average AUC was above 0.75. Higher AUC values correlate with lower $p$-values, with the exception of the "old" task. For this task, the AUC is minimum compared to the other tasks, while the $p$-value brain vs. random is on the lower end again in comparison.

\section{DISCUSSION}

The objective of our research was to study whether image generation process could be directed solely based on feedback obtained via brain-computer interfacing. That is, whether brain feedback could be used in adjusting a generative model output by means of a user mentally detecting features. Brain feedback was obtained via EEG-based Brain-Computer In- terfacing and allowed implicit detection of user intentions without requiring any explicit feedback.

\section{Contributions}

In order to study whether image generation according to user intentions is possible via brain feedback and how it performs, we asked three research questions. Here, we discuss the results accordingly.

RQ1: Can human brain responses be utilized as feedback to a generative model to produce task-relevant images?

A1: Our results show that participants recognize the intended task relevant features, the brain signals evoked by recognition can be reliably classified and fed as feedback to the generative model. The intended task relevant features are also modeled by the GAN model and brain feedback on the GAN model produces images that match the mental image categories instructed to be the targets of the participants. Simulations with combined tasks also show high performance even when three different target features are provided simultaneously.

RQ2: How fast and well does brain feedback converge compared to explicit and random feedback?

A2: Brain feedback shows consistently increasing performance as a function of the feedback iterations. The image quality improves rapidly only after $1-5$ feedback iterations and saturates around 10-15 iterations. The brain feedback performs significantly better than random feedback and the quality of the generated images is indistinguishable from the output of an explicit feedback process. Our results indicate that brain feedback can have high utility in practical applications and reaches performance that is comparable to explicit manual feedback.

RQ3: How well does brain feedback converge on multiple simultaneous task targets?

A3: Our results show that brain feedback leads to high quality task-relevant images, also in cases where many individual goals are combined in a single generative process. Such 

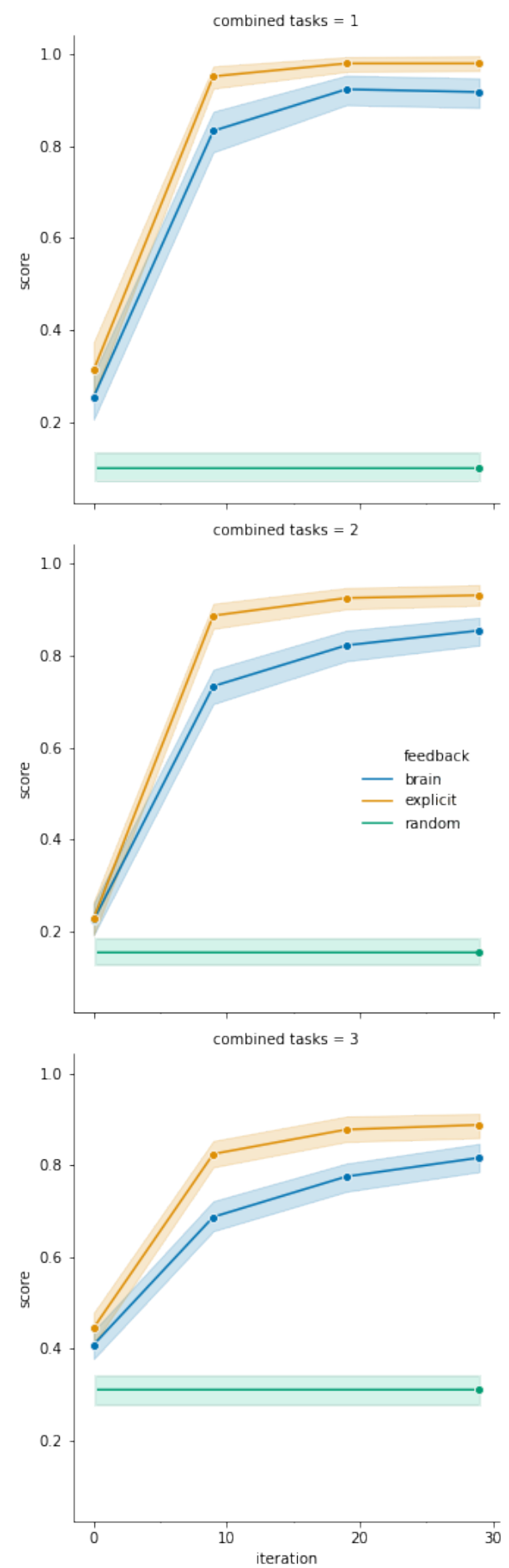

Figure 4. Quality score over feedback iterations for brain feedback, explicit feedback, and random feedback (mean over the tasks/task combinations and participants). The results are shown as a mean of single tasks (top), a mean of combinations of two tasks (middle), and a mean of combinations of three tasks (bottom).

multi-aspect task combinations are modeled remarkably well and converge equally fast as the sigle task processes.
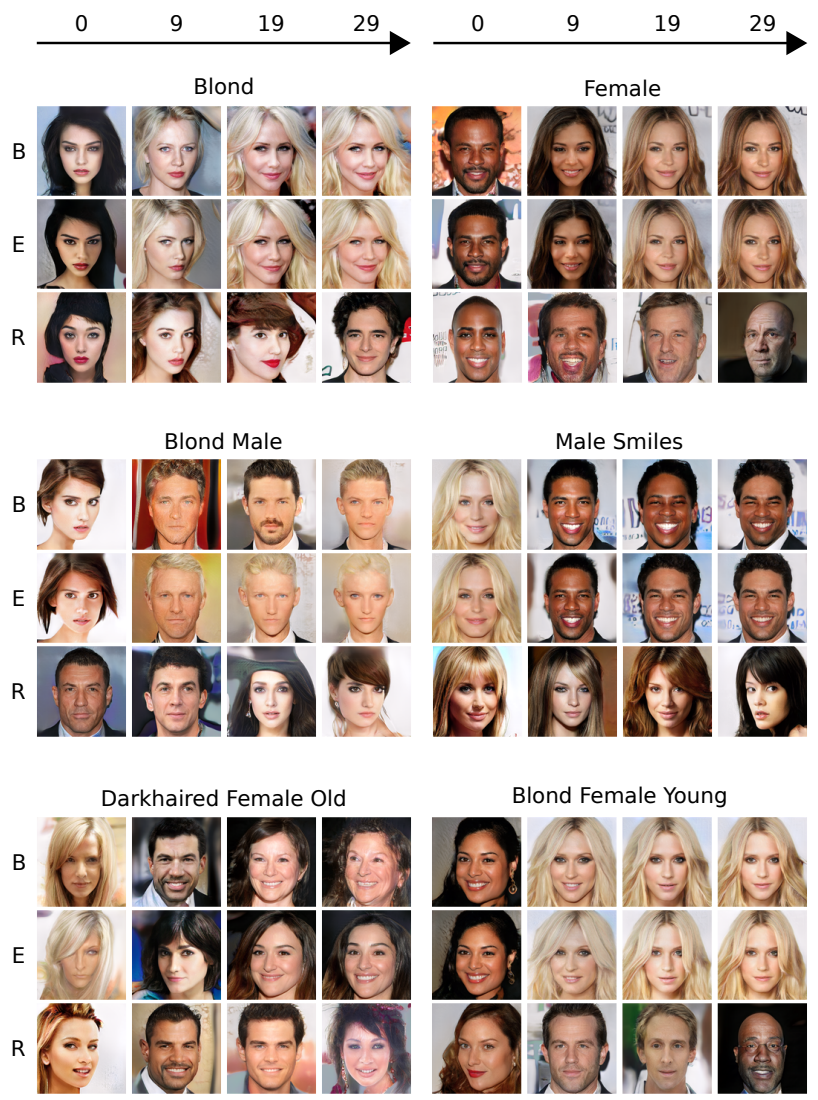

Figure 5. Convergence of generated images at iterations $0,9,19$, and 29, for brain (B), explicit (E), and random (R) feedback.

\section{Limitations and future work}

Currently, our approach exploits a relatively restricted GAN model that is trained with images from a single and narrow domain of celebrity faces. A practically useful application of our approach beyond face images would have to be trained with a large and diverse image data, which might lead to more complex model and different performance than shown in the present experiments.

The EEG data is recorded in a laboratory setting, which is designed to maximize signal-to-noise ratio and minimize artefacts, such as movements or natural, but disturbing competing stimuli. Real-world applications of our approach would require experimentation in less restricted settings and possibly with more comfortable wearable devices.

The task combinations were sampled to avoid combinatorial explosion of the generated output images. While the sampling was fully randomized, not all possible task combinations were evaluated in the experiments. Generally, our experiments show constant performance, but more experimentation is needed to ensure the generalizability of our findings with greater variance of data, models, and tasks. 
Our computational approach could be extended to machine learning architectures that could jointly model the classification and GAN models, instead of relying on separate ERP classifier and generative model. Unsupervised approaches could also be explored to determine evoked brain responses without a reliance on labeled data.

Computational requirements of image generation with GANs prevented us from creating a closed-loop brain-computer interface. However, the present study shows the feasibility of such system by introducing all necessary components to guide a future implementation.

\section{CONCLUSIONS}

We set out to study if it is feasible to combine an image generation process based on a neural network with brain feedback. Our approach was implemented as an interaction between a state-of-the-art generative adversarial neural network and an EEG based brain-computer interface trained to detect relevance signal on the images generated by the neural network. Our experiments show that our approach allowed generating relevant images matching the given tasks, and the brain feedback yielded a performance close to a comparison condition where explicit manual feedback was used. The presented results open avenues for connecting brain-computer interfaces with neural networks to augment human-computer interaction for complex, yet realistic, creative tasks. This system requires no physical interaction and only minimal workload, while achieving the same performance as one based on explicit feedback. The presented paradigm enables us to develop BCI applications for more complex tasks, making possible to augment creative processes with technologies for implicit interaction and human-AI collaboration. Despite the advantages and impressive achievements of generative models, these techniques may also have potentially harmful applications. Generating images provokes problems of fake, or unreal content, to be generated and exploited in possibly precarious ways. The present research aimed to study whether directing generative models using brain responses would be possible in the first place. However, as this process is directed implicitly, or even unconsciously via brain-computer interfacing, ethical concerns may arise and require investigation whenever such techniques are put in practical use.

\section{ACKNOWLEDGEMENTS}

We would like to thank Esther Ortiz for providing user assessments. The research was partially funded by the Academy of Finland. Computing resources were provided by the Finnish Grid and Cloud Infrastructure (persistent id: urn:nbn:fi:research-infras-2016072533).

\section{REFERENCES}

[1] Emanuel Donchin and Michael GH Coles. 1988. Is the P300 component a manifestation of context updating? Behavioral and brain sciences 11, 03 (1988), 357-374.

[2] Emanuel Donchin, Kevin M Spencer, and Ranjith Wijesinghe. 2000. The mental prosthesis: assessing the speed of a P300-based brain-computer interface. IEEE transactions on rehabilitation engineering 8, 2 (2000), 174-179.
[3] Manuel JA Eugster, Tuukka Ruotsalo, Michiel M Spapé, Ilkka Kosunen, Oswald Barral, Niklas Ravaja, Giulio Jacucci, and Samuel Kaski. 2014. Predicting term-relevance from brain signals. In Proc. SIGIR. ACM, 425-434.

[4] Manuel J. A. Eugster, Tuukka Ruotsalo, Michiel M. Spapé, Oswald Barral, Niklas Ravaja, Giulio Jacucci, and Samuel Kaski. 2016. Natural brain-information interfaces: Recommending information by relevance inferred from human brain signals. Scientific Reports 6 (Dec. 2016), 38580.

[5] Hao Fang, Saurabh Gupta, Forrest Iandola, Rupesh K. Srivastava, Li Deng, Piotr Dollar, Jianfeng Gao, Xiaodong He, Margaret Mitchell, John C. Platt, C. Lawrence Zitnick, and Geoffrey Zweig. 2015. From Captions to Visual Concepts and Back. In Proc. CVPR.

[6] Lawrence Ashley Farwell and Emanuel Donchin. 1988. Talking off the top of your head: toward a mental prosthesis utilizing event-related brain potentials. Electroencephalography and clinical Neurophysiology 70, 6 (1988), 510-523.

[7] P.I. Good. 2000. Permutation Tests: A Practical Guide to Resampling Methods for Testing Hypotheses (2nd ed.). Springer.

[8] Ian J. Goodfellow, Jean Pouget-Abadie, Mehdi Mirza, Bing Xu, David Warde-Farley, Sherjil Ozair, Aaron C. Courville, and Yoshua Bengio. 2014. Generative Adversarial Nets. In Proc. NIPS. 2672-2680.

[9] Longteng Guo, Jing Liu, Yuhang Wang, Zhonghua Luo, Wei Wen, and Hanqing Lu. 2017. Sketch-Based Image Retrieval Using Generative Adversarial Networks. In Proc. MM. Association for Computing Machinery, New York, NY, USA, 1267-1268.

[10] Giulio Jacucci, Oswald Barral, Pedram Daee, Markus Wenzel, Baris Serim, Tuukka Ruotsalo, Patrik Pluchino, Jonathan Freeman, Luciano Gamberini, Samuel Kaski, and others. 2019. Integrating neurophysiologic relevance feedback in intent modeling for information retrieval. Journal of the Association for Information Science and Technology 70, 9 (2019), 917-930.

[11] Lauri Kangassalo, Michiel Spapé, Giulio Jacucci, and Tuukka Ruotsalo. 2019. Why Do Users Issue Good Queries? Neural Correlates of Term Specificity. In Proc. SIGIR. ACM, New York, NY, USA, 375-384.

[12] Lauri Kangassalo, Michiel Spapé, Niklas Ravaja, and Tuukka Ruotsalo. 2020. Information gain modulates brain activity evoked by reading. Scientific reports 10,1 (2020), 1-10.

[13] Lauri Kangassalo, Michiel M. Spapé, and Tuukka Ruotsalo. 2020. Neuroadaptive modelling for generating images matching perceptual categories. Scientific Reports (2020), to appear. 
[14] Tero Karras, Timo Aila, Samuli Laine, and Jaakko Lehtinen. 2018. Progressive Growing of GANs for Improved Quality, Stability, and Variation. In Proc. ICLR.

[15] Tero Karras, Samuli Laine, and Timo Aila. 2019. A Style-Based Generator Architecture for Generative Adversarial Networks. In Proc. CVPR. 4401-4410.

[16] Isaak Kavasidis, Simone Palazzo, Concetto Spampinato, Daniela Giordano, and Mubarak Shah. 2017. Brain2Image: Converting Brain Signals into Images. In Proc. MM. ACM, New York, NY, USA, 1809-1817.

[17] Boris Kotchoubey. 2006. Event-related potentials, cognition, and behavior: a biological approach. 30, 1 (2006), 42-65.

[18] Ren Li, Jared S Johansen, Hamad Ahmed, Thomas V Ilyevsky, Ronnie B Wilbur, Hari M Bharadwaj, and Jeffrey Mark Siskind. 2018. Training on the test set? An analysis of Spampinato et al.[arXiv: 1609.00344]. arXiv preprint arXiv:1812.07697 (2018).

[19] Z. Liu, P. Luo, X. Wang, and X. Tang. 2015. Deep Learning Face Attributes in the Wild. In Proc. ICCV. 3730-3738.

[20] Yongyi Lu, Shangzhe Wu, Yu-Wing Tai, and Chi-Keung Tang. 2018. Image Generation from Sketch Constraint Using Contextual GAN. In Proc. ECCV.

[21] Markus Ojala and Gemma C. Garriga. 2009. Permutation Tests for Studying Classifier Performance. In Proc. Data Mining. IEEE, Miami Beach, FL, USA, 908-913.

[22] Terence W Picton. 1992. The P300 wave of the human event-related potential. Journal of clinical neurophysiology 9, 4 (1992), 456-479.

[23] John Polich. 2007. Updating P300: an integrative theory of P3a and P3b. Clinical neurophysiology 118, 10 (2007), 2128-2148.

[24] J. J. Rocchio. 1971. Relevance Feedback in Information Retrieval. Prentice Hall, Englewood, Cliffs, New Jersey.

[25] Patsorn Sangkloy, Jingwan Lu, Chen Fang, Fisher Yu, and James Hays. 2017. Scribbler: Controlling Deep Image Synthesis With Sketch and Color. In Proc. CVPR.

[26] Concetto Spampinato, Simone Palazzo, Isaak Kavasidis, Daniela Giordano, Nasim Souly, and Mubarak Shah. 2017. Deep Learning Human Mind for Automated Visual Classification. In Proc. CVPR.

[27] Michiel Spapé, Rinus Verdonschot, and Henk van Steenbergen. 2019. The E-Primer: An introduction to creating psychological experiments in E-Prime. Second edition updated for E-Prime 3. (2 ed.). Leiden Univerisity Press.

[28] Nancy K Squires, Kenneth C Squires, and Steven A Hillyard. 1975. Two varieties of long-latency positive waves evoked by unpredictable auditory stimuli in man. Deux varieties d'ondes positives de longue latence evoquees par des stimuli auditifs non predictibles chez l'homme. Electroencephalography and Clinical Neurophysiology 38, 4 (1975), 387-401.

[29] Samuel Sutton, Patricia Tueting, Joseph Zubin, and E. Roy John. 1967. Information delivery and the sensory evoked potential. Science 155, 3768 (1967), 1436-1439.

[30] Praveen Tirupattur, Yogesh Singh Rawat, Concetto Spampinato, and Mubarak Shah. 2018. ThoughtViz: Visualizing Human Thoughts Using Generative Adversarial Network. In Proc. MM. ACM, New York, NY, USA, 950-958.

[31] Anne M Treisman and Garry Gelade. 1980. A feature-integration theory of attention. Cognitive psychology 12, 1 (1980), 97-136.

[32] Antti Ukkonen, Pyry Joona, and Tuukka Ruotsalo. 2020. Generating Images Instead of Retrieving Them: Relevance Feedback on Generative Adversarial Networks. In Proc. SIGIR. ACM, New York, NY, USA, 1329-1338.

[33] Rolf Verleger, Piotr Jaśkowski, and Edmund Wascher. 2005. Evidence for an integrative role of P3b in linking reaction to perception. Journal of Psychophysiology 19, 3 (2005), 165-181.

[34] Kelvin Xu, Jimmy Lei Ba, Ryan Kiros, Kyunghyun Cho, Aaron Courville, Ruslan Salakhutdinov, Richard S. Zemel, and Yoshua Bengio. 2015. Show, Attend and Tell: Neural Image Caption Generation with Visual Attention. In Proc. ICML. JMLR.org, 2048-2057.

[35] Thorsten O Zander, Laurens R Krol, Niels P Birbaumer, and Klaus Gramann. 2016. Neuroadaptive technology enables implicit cursor control based on medial prefrontal cortex activity. Proceedings of the National Academy of Sciences 113, 52 (2016), 14898-14903.

[36] Han Zhang, Tao Xu, Hongsheng Li, Shaoting Zhang, Xiaogang Wang, Xiaolei Huang, and Dimitris N. Metaxas. 2017. StackGAN: Text to Photo-Realistic Image Synthesis With Stacked Generative Adversarial Networks. In Proc. ICCV.

[37] Xiang Sean Zhou and Thomas S Huang. 2003. Relevance feedback in image retrieval: A comprehensive review. Multimedia systems 8, 6 (2003), 536-544. 


\begin{tabular}{|c|c|c|c|c|c|c|c|}
\hline & Random $F B \pm \sigma$ & Brain $F B \pm \sigma$ & Explicit $F B \pm \sigma$ & $(F) B v s . R$ & $(F) B$ vs. $E$ & $\Delta B$ vs. $R$ & $\Delta B$ vs. $E$ \\
\hline \multicolumn{8}{|l|}{ Single tasks } \\
\hline Blond & $0.04 \pm 0.21$ & $0.92 \pm 0.26$ & $1.00 \pm 0.00$ & 175.28 & 2.95 & $+87.59^{* * *}$ & -8.06 \\
\hline Darkhaired & $0.03 \pm 0.12$ & $0.93 \pm 0.25$ & $1.00 \pm 0.00$ & 324.41 & 2.07 & $+90.32^{* * *}$ & -6.45 \\
\hline Female & $0.06 \pm 0.25$ & $1.00 \pm 0.00$ & $1.00 \pm 0.00$ & 435.00 & 0.00 & $+93.55^{* * *}$ & 0.00 \\
\hline Male & $0.03 \pm 0.18$ & $0.93 \pm 0.25$ & $1.00 \pm 0.00$ & 267.27 & 2.07 & $+90.32^{* * *}$ & -6.45 \\
\hline Nosmile & $0.17 \pm 0.35$ & $0.90 \pm 0.30$ & $0.97 \pm 0.18$ & 75.56 & 1.05 & $+72.58^{* * *}$ & -6.45 \\
\hline Old & $0.11 \pm 0.28$ & $0.92 \pm 0.24$ & $0.97 \pm 0.18$ & 154.95 & 0.86 & $+80.64^{* * *}$ & -4.84 \\
\hline Smiles & $0.10 \pm 0.30$ & $0.89 \pm 0.31$ & $0.97 \pm 0.18$ & 104.49 & 1.58 & $+79.03^{* * *}$ & -8.06 \\
\hline Young & $0.22 \pm 0.31$ & $0.84 \pm 0.35$ & $0.93 \pm 0.25$ & 55.41 & 1.56 & $+62.00^{* * *}$ & -9.68 \\
\hline Mean & $0.10 \pm 0.25$ & $0.92 \pm 0.24$ & $0.98 \pm 0.10$ & 199.05 & 1.52 & $+82.00^{* * *}$ & -6.25 \\
\hline \multicolumn{8}{|c|}{ Sample of combinations of two tasks } \\
\hline Blond, male & $0.13 \pm 0.22$ & $0.82 \pm 0.24$ & $0.97 \pm 0.12$ & 137.30 & 8.74 & $+69.35^{* * *}$ & $-14.52^{*}$ \\
\hline Blond, old & $0.25 \pm 0.31$ & $0.92 \pm 0.19$ & $0.98 \pm 0.09$ & 98.72 & 3.01 & $+66.67^{* * *}$ & -6.67 \\
\hline Blond, young & $0.08 \pm 0.19$ & $0.85 \pm 0.26$ & $0.93 \pm 0.17$ & 177.23 & 2.04 & $+77.42^{* * *}$ & -8.06 \\
\hline Darkhaired, male & $0.05 \pm 0.15$ & $0.95 \pm 0.20$ & $1.00 \pm 0.00$ & 409.04 & 1.85 & $+90.32^{* * *}$ & -4.84 \\
\hline Female, young & $0.10 \pm 0.24$ & $0.98 \pm 0.09$ & $1.00 \pm 0.00$ & 375.00 & 1.00 & $+88.71^{* * *}$ & -1.61 \\
\hline Male, nosmile & $0.10 \pm 0.20$ & $0.89 \pm 0.21$ & $0.97 \pm 0.12$ & 226.51 & 3.32 & $+79.03^{* * *}$ & -8.06 \\
\hline Male, smiles & $0.10 \pm 0.20$ & $0.82 \pm 0.30$ & $0.97 \pm 0.12$ & 122.97 & 6.04 & $+72.58^{* * *}$ & -14.52 \\
\hline Male, young & $0.42 \pm 0.26$ & $0.60 \pm 0.30$ & $0.63 \pm 0.22$ & 6.15 & 0.23 & $+17.74^{*}$ & -3.22 \\
\hline Mean & $0.15 \pm 0.22$ & $0.85 \pm 0.22$ & $0.93 \pm 0.11$ & 194.11 & 3.28 & $+70.23^{*}$ & -7.70 \\
\hline \multicolumn{8}{|c|}{ Sample of combinations of three tasks } \\
\hline Blond, female, smiles & $0.16 \pm 0.24$ & $0.92 \pm 0.19$ & $0.97 \pm 0.12$ & 194.91 & 1.44 & $+75.81^{* * *}$ & -4.84 \\
\hline Blond, female, young & $0.11 \pm 0.21$ & $0.93 \pm 0.17$ & $1.00 \pm 0.00$ & 282.71 & 4.44 & $+82.26^{* * *}$ & -6.45 \\
\hline Blond, male, nosmile & $0.26 \pm 0.25$ & $0.74 \pm 0.25$ & $0.89 \pm 0.21$ & 56.25 & 5.95 & $+48.39^{* * *}$ & -14.52 \\
\hline Darkhaired, female, old & $0.57 \pm 0.17$ & $0.75 \pm 0.25$ & $0.85 \pm 0.23$ & 10.66 & 2.52 & $+18.33^{*}$ & -10.00 \\
\hline Darkhaired, male, young & $0.27 \pm 0.25$ & $0.66 \pm 0.24$ & $0.76 \pm 0.25$ & 28.57 & 2.40 & $+38.71^{* * *}$ & -9.68 \\
\hline Female, nosmile, old & $0.45 \pm 0.24$ & $0.73 \pm 0.25$ & $0.73 \pm 0.25$ & 19.72 & 0.00 & $+28.33^{* * *}$ & 0.00 \\
\hline Female, old, smiles & $0.43 \pm 0.17$ & $0.88 \pm 0.21$ & $0.90 \pm 0.20$ & 79.78 & 0.09 & $+45.00^{* * *}$ & -1.67 \\
\hline Male, nosmile, old & $0.23 \pm 0.31$ & $0.90 \pm 0.20$ & $1.00 \pm 0.00$ & 95.08 & 7.25 & $+66.67^{* * *}$ & -10.00 \\
\hline Mean & $0.31 \pm 0.23$ & $0.82 \pm 0.22$ & $0.90 \pm 0.16$ & 95.96 & 3.01 & $+50.44^{* *}$ & -7.14 \\
\hline
\end{tabular}

Table 2. The overall quality of generated images for all individual models, combined models, and feedback modalities (R: random; B: brain; E: explicit). The results are shown with standard deviation, improvement in image quality between the baseline and feedback methods $(\Delta)$, and Bonferroni corrected $p$-values and the respective $F$-statistics. 